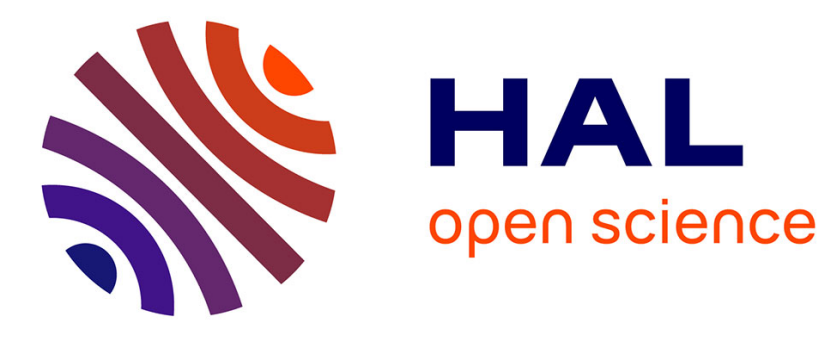

\title{
Eco-efficient management of a feeding system in an automobile assembly-line
}

Montserrat-Ana Miranda, María Jesús Alvarez, Cyril Briand, Matías Urenda Moris, Victoria Rodríguez

\section{- To cite this version:}

Montserrat-Ana Miranda, María Jesús Alvarez, Cyril Briand, Matías Urenda Moris, Victoria Rodríguez. Eco-efficient management of a feeding system in an automobile assembly-line. Journal of Modelling in Management, 2020, ahead-of-print, 10.1108/jm2-10-2019-0241 . hal-03010898

\section{HAL Id: hal-03010898 \\ https://hal.science/hal-03010898}

Submitted on 17 Nov 2020

HAL is a multi-disciplinary open access archive for the deposit and dissemination of scientific research documents, whether they are published or not. The documents may come from teaching and research institutions in France or abroad, or from public or private research centers.
L'archive ouverte pluridisciplinaire HAL, est destinée au dépôt et à la diffusion de documents scientifiques de niveau recherche, publiés ou non, émanant des établissements d'enseignement et de recherche français ou étrangers, des laboratoires publics ou privés. 


\title{
Eco-efficient management of a feeding system in an automobile assembly-line
}

\author{
Montserrat-Ana Miranda \\ Department of Business Administration, School of Economics and \\ Business Administration, University of Navarra, Pamplona, Spain \\ María Jesús Alvarez \\ Department of Industrial Organization, School of Engineering (TECNUN), \\ University of Navarra, San Sebastián, Spain \\ Cyril Briand \\ Department of Decision and Optimization, Laboratory of Analysis and \\ Architecture of Systems (LAAS-CNRS), Toulouse, France \\ Matías Urenda Moris \\ Department of Engineering Sciences, Uppsala University, Uppsala, Sweden, and \\ Victoria Rodríguez \\ Department of Business Administration, School of Economics and \\ Business Administration, University of Navarra, Pamplona, Spain
}

\begin{abstract}
Purpose - This study aims to reduce carbon emissions and costs in an automobile production plant by improving the operational management efficiency of a serial assembly line assisted by a feeding electric tow vehicle (ETV).

Design/methodology/approach - A multi-objective function is formulated to minimize the energy consumption of the ETV from which emissions and costs are measured. First, a mixed-integer linear programming model is used to solve the feeding problem for different sizes of the assembly line. Second, a biobjective optimization (HBOO) model is used to simultaneously minimize the most eco-efficient objectives: the number of completed runs (tours) by the ETV along the assembly line, and the number of visits (stops) made by the ETV to deliver kits of components to workstations.
\end{abstract}

Findings - The most eco-efficient strategy is always the bi-objective optimal solution regardless of the size of the assembly line, whereas, for single objectives, the optimization strategy differs depending on the size of the assembly line.

Research limitations/implications - Instances of the problem are randomly generated to reproduce real conditions of a particular automotive factory according to a previous case study. The optimization procedure allows managers to assess real scenarios improving the assembly line eco-efficiency. These results promote the implementation of automated control of feeding processes in green manufacturing.

Originality/value - The HBOO-model assesses the assembly line performance with a view to reducing the environmental impact effectively and contributes to reducing the existent gap in the literature. The

Authors acknowledge support from the European Comission ECO-INNOVERA project: EASY (Energy Aware feeding SYstems) and from the Spanish Government project PRI-PIMEIN-2011-1465 for supporting international collaboration in green technologies and services.

Eco-efficient management

Received 8 October 2019 Revised 19 February 2020 17 May 2020 Accepted 12 June 2020




optimization results define key strategies for manufacturing industries eager to integrate battery-operated motors or to address inefficient traffic of automated transport to curb the carbon footprint.

Keywords Optimization, Energy efficiency, Scheduling, Supply chain management,

Ecological efficiency, Green assembly line operational management,

Carbon footprint, Bi-objective optimization, Assembly line scheduling,

Sustainable supply chain management

Paper type Research paper

\section{Introduction and motivation}

Recent concerns over eco-efficiency have turned assembly line innovation toward sustainability and cost reduction into primary goals in manufacturing industries such as the automotive, aerospace, civil engineering and renewable energy industries. Optimization of assembly lines performance is of primary interest in manufacturing industries where balancing and scheduling problems represent the main leading research areas (Fathi et al., 2018; Scholl et al., 2013; Andres et al., 2008; Boysen et al., 2007). To this respect, the present work is developed within the framework of the later, but under energy efficiency concerns.

Generally, an assembly line involves several elements such as a warehousing space or supermarket, a forward distribution of serial and paced stations where specific operations are performed on products, a conveyor belt and a feeding tow vehicle (or a fleet) which refills the inventory at stations attended by working operators (workstations). Still, on a larger scale, assembly lines take part in supply chain networks whose optimization problems are undergoing a transformation along with higher demands in competitiveness and higher degrees of interaction between manufacturers, suppliers and costumers (Gharaei et al., 2017). As well, there is a higher commitment to reduce environmental impact (Kähkönen, 2020; Das and Jharkharia, 2018; Alexander et al., 2014; Srivastava, 2007). Howbeit, optimization of processes to detect energy inefficiencies in the supply chain and inventory management are still to face new challenges as pointed by previous works (Wang et al., 2016; Bazan et al., 2015; Benjaafar et al., 2013).

According to the International Energy Agency (IEA, 2007) by adopting eco-efficient policies, which imply both, economic and energy efficiency measures to reduce the environmental impact, industrial $\mathrm{CO}_{2}$ emissions can be reduced by $7 \%-12 \%$ of today's global $\mathrm{CO}_{2}$ emissions (1.9 to $3.2 \mathrm{Gt}$ per year). Notably, in the manufacturing industry, motordriven systems (engines) account for $15 \%$ of the final energy consumed in manufacturing. In this regard, the IEA advocates to particularize the estimation of energy efficiency for each type of engine expecting energy savings up to $60 \%$ when the velocity of the engines is controlled.

In this direction, a sustainable supply chain management must integrate eco-efficient protocols affecting:

- The distribution operations from the suppliers to the supermarket (SM) of the industrial plant.

- The feeding problem in the assembly line.

- The transport logistics from the manufacturer to the retailer.

- The present work refers to the second item mentioned above, aiming at reducing the carbon footprint in a car assembly line, which produces the end-product.

The model of study is a serial assembly line that starts and ends at the SM like a unidirectional closed-loop. In addition, a deterministic production rate, which is planned 
over a daily time horizon, is considered. This deterministic model involves fixed demands and fixed capacities at workstations which are predetermined by assembly-line production processes. Equivalent $\mathrm{CO}_{2}\left(\mathrm{CO}_{2}-\mathrm{eq}\right)$ emissions and economic costs are calculated from the energy consumption of an electric tow vehicle (ETV), which feeds the workstations along the assembly line. A global review on environmental management in the automotive industry can be found in (Liu et al., 2016).

To assess eco-efficient actions in the automotive plant, the energy recovery system of an ETV, known as the regenerative braking effect, is integrated into our model. As reported by $\mathrm{Xu}$ et al. (2011) and Jin et al. (2014) and references therein, energy efficiency due to regenerative braking has shown to be up to roughly $30 \%$ which represents an average value if the origin of the electricity source (renewable energy or fossil fuel) is disregarded. It must be emphasized that, although the energy consumption of a single ETV accounts for a small fraction of the total energy demanded by an automobile assembly line, it is crucial to model and analyze every aspect in the assembly line management to assume a realistic commitment to reduce $\mathrm{CO}_{2}$ emissions.

Because of the nonlinear nature of the function defining the energy consumption, the feeding problem in this study is approached from a different perspective which considers the linear combination of those objectives which are concurrently related to the energy consumption in the form of a multi-objective optimization problem. Weighting coefficients are assigned to each objective component to express their relative importance. Multiobjective linear programing problems (Figueira et al., 2005; Sawaragi et al., 1985) subjected to a hierarchical order of restrictions over its variables have been successfully implemented in operational research, management sciences and engineering design and control problems to optimize single or more objectives simultaneously. Multi-objective optimization in management is of utmost importance because results often surpass prior assumptions bringing into light new strategies for decision-makers.

In accordance with the objective function and constraints of our problem, we propose a mixed-integer linear programming (MILP) problem. The MILP-problem includes the following objectives: the number of completed runs driven by the ETV along the entire assembly line (tours), the number of visits to feed the workstations (stops) and the inventory level at workstations (inventory). A branch-and-cut algorithm, which combines the searchtree and the cutting-planes method (CPLEX [1]), is used to solve the MILP-problem. On the one hand, the branch-and-cut algorithm has shown to be powerful in similar problems like vehicle routing problems (Araque et al., 1994; Archetti et al., 2007; Rodríguez et al., 2007; Barcos et al., 2010) and assembly line feeding problems (Fathi et al., 2014a, 2014b). On the other hand, the CPLEX optimizer allows us to codify the MILP-problem and solve it within the MATLAB environment where a downstream code quantifies the carbon footprint from energy consumption. The CPLEX optimization solver is advantageous for defining binary indicator variables which are of critical importance in routing optimization (Bonami et al., 2015). Moreover, optimization parameters in the branch-and-cut-algorithm such as gap parameters for the populate procedure, tolerance parameters for the minimization problem and constraint relaxation preferences can be tuned to control the reliability of the results which, in our study, are ultimately tested against technical reports concerning the energy consumption and emissions of electric vehicles. We find previous reports that compare the CPLEX performance against other solvers in routing problems (Shijin and Yulun, 2015; Yu and Jewpanya, 2016; Bonami et al., 2015), and further research on CPLEX performance is still being developed to boost computational experiments (Bonami et al., 2019). Furthermore, it should be noted that there is a great deal of research works in routing vehicle problems and green logistics that have been implementing CPLEX successfully (Keskin et al., 2019; 
Huang and Yang, 2019; Bierwirth et al., 2019; Azmi et al., 2017; Petridis et al., 2017; Wassan et al., 2017; Salhi and Imran, 2014; Kopfer and Kopfer, 2013; Toth, 2008), just to mention a few.

Because of the fact that the two most conflicting objectives are the number of tours and stops because of their competing levels of energy consumption as the size of the assembly line increases, we show that the problem needs to be addressed using a different method, and hence, a hybrid bi-objective optimization (HBOO) method is proposed. Bi-objective optimization problems generally involve dependent objectives with competing roles, and thus, solutions may not show identical degrees of optimality with regard to both objectives, simultaneously; as a consequence, it is necessary to implement a lexicographic optimization method (Ehrgott, 2005). In particular, the HBOO-model has been designed to follow a lexicographic weighted Tchebycheff approach (Steuer and Choo, 1983; Dächert et al., 2012). Fundamentally, a lexicographic optimization method selects the best solution with respect to a particular objective function given the fact that the other objective function has multiple optimal solutions. The HBOO-method runs in two different phases. In the first phase, a Pareto front is built from sets of optimal solutions that define a convex border that represents the trade-off between the two dependent objectives. As a matter of fact, Pareto fronts inform decision-makers about the best compromise solution. In this respect, the solution that effectively satisfies the minimum value of the bi-objective function is expected to be localized at the turning point, which is referred to as the knee solution of this convex Pareto front (Emmerich and Deutz, 2007; Setämaa-Kärkkäinen et al., 2006). In fact, the knee solution represents the most convenient bi-objective solution and the one at which the HBOO-problem is targeted in the second phase. Be that as it may, the HBOO-method allows top managers to have access to each phase separately to assess strategic decisionmaking, and thus, it is a flexible model that promotes a gradual implementation of new operational logistics planning toward a sustainable manufacturing scenario.

To understand inherent dependencies between two different objectives, such as the case of objectives (tours) and (stops) in the present study, it is necessary to review here the concept of dominance between two different solutions $u$ and $v$ (vectors of decision variables) that belong to the feasible set of solutions $S$ of a bi-objective optimization problem. Being $x$ the vector of decision variables, and $f(x)=f_{1}(x)+f_{2}(x)$ the bi-objective function where $f_{1}(x)$ and $f_{2}(x)$ represent the objectives to be minimized simultaneously. Now, given two solution vectors $u$ and $v$ that optimize $f(x)$, we say that $u$ dominates $v$ if $f_{i}(u) \leq f_{i}(v) \forall i$ (where $i$ is the number of objective functions, $i=2$ ) except for at least one $i=j$ for which $f_{j}(u)<f_{j}(v)$. According to this, a vector of objective variables $w \in S$ is said to be Pareto optimal if it is not dominated by any other solution vector, then we say that the Pareto optimal set consists of non-dominated solutions. In other words, when it comes to solving a bi-objective optimization problem, it is impossible to improve the optimality of an objective without jeopardizing the optimality of the other objects in the solution vector. In terms of solution vectors, it can be rigorously defined if we say that a decision vector $u \in S$ and the corresponding objective vector $f(u)$ are weak Pareto optimal if there is not another decision vector $v \in S$ such that $f_{i}(u)<f_{i}(v)$ for $i=1,2$ (Sawaragi et al., 1985).

Prior studies on bi-objective optimization methods applied to supply chain management can be found to design green automotive supply chains (Zhen, 2017; Sadrnia et al., 2013) and to solve location-allocation problems (Alavidoost et al., 2018; Latha Shankar et al., 2012). Nevertheless, bi-objective optimization procedures applied to assembly line scheduling problems with eco-efficiency criteria are still scarce (Zhou and Shen, 2018), and for this reason, the present work intends to address this gap in literature proposing a new optimization method. 
The main application of our research is to help managers to evaluate feeding processes in manufacturing plants toward sustainability. Furthermore, beyond the discovery and analysis of the operational management factors that have a critical role in the green assembly line performance, the optimization results also provide decision support with new strategies to be implemented in automated feeding processes. To assess the eco-efficient management of the assembly line, we evaluate emissions and costs from the optimal solutions for which different velocity profiles of the ETV are tested. In addition, the regenerative braking effect of the ETV as a way of recapturing energy is also tested. To further support these results, we show that energy savings are in agreement with previous studies.

The present paper is organized as follows, in Section 2 we present the particularities of our automobile assembly line, the emissions assessment and the generation of instances. In Section 3, we show how the problem is formulated as a MILP-program, and the HBOOmodel. Results on emissions and costs for different sizes of the assembly line are presented in Section 4. In addition, in this section, the optimality of the results are tested against standard values. Results are interpreted in terms of new strategies to be implemented in automated assembly lines to reduce the environmental impact, and to increase the operational management efficiency. Finally, Section 5 deals conclusively with the most remarkable results and future perspectives.

\section{Problem statement}

In this section, we deal with the emissions assessment of the ETV and the generation of instances. The assembly line consists of $N$-workstations separated by a fixed inter-spacing, $d$, as it is sketched in Figure 1(a), where an ETV feeds the assembly line through different runs or tours which extend over the planning horizon of a single day. In this study, we present results for different sizes of the assembly line consisting of $N=20,40$ and 60 workstations. Distances between contiguous workstations are fixed to $d=5 \mathrm{~m}$. Similar factory conditions are found in previous scheduling problems in the automobile industry (Fathi et al., 2014a, 2014b; Muguerza et al., 2015). In a previous work where we had tested sizes of the assembly line for a wider range and different values of $d$, it was found that results on $N=20$ are sufficiently representative of smaller sizes of the assembly line while results on $N=60$ are sufficiently representative of larger sizes. On the other side, by increasing the value of parameter $d$ results in emissions (costs) increase proportionally as

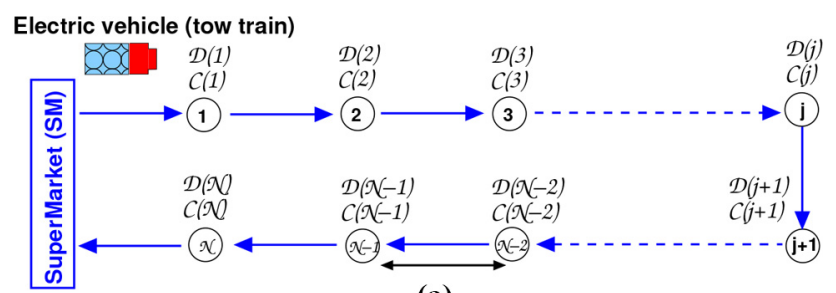

(a)

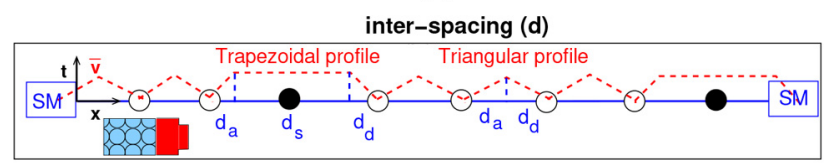

(b) Eco-efficient
management

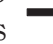


expected from the energetic considerations of the problem as it is explained in the next Section 2.1. The SM is the logistics storage area where bins, which are the kits of components specifically packed for different workstations, are stacked while waiting to be loaded onto the trailers of an ETV, a Linde P60z tow vehicle with a nominal towing capacity of 6 tonnes. Two trailers are hauled by the ETV. Distances from the SM to the first workstation, and identical than from the last workstation to the SM $(d=5 \mathrm{~m})$. Bins are delivered to each workstation according to its storage capacity (inventory level of each workstation), $C(j)$ and daily demand, $D(j)$. We have chosen a mixed-linear optimization model to address the scheduling problem in which units of kits are delivered to the line from and back to the SM along each tour.

The ETV performs two possible types of velocity profiles which are defined for simplicity as triangular and trapezoidal as it is shown in Figure 1(b). In this figure, planned stops are represented by non-filled circles while skipped stops (not visited workstations) are filled in black. Triangular-like velocity profiles are triggered for consecutive stops below a threshold distance of $10 \mathrm{~m}$, where the engine accelerates until reaching a mid-path position and then decelerates to the next stop. Above the threshold distance, the trapezoidal velocity profile is triggered, then the ETV accelerates to reach the maximum speed and then decelerates to the next stop.

\subsection{Emissions assessment}

We assume that the carbon footprint is given by indirect emissions due to the electricity consumed by the ETV battery. The energy consumption of the engine battery (Lee, 2005; Helmers and Marx, 2012) is quantified so that the traction power from the driveline accounts for the net battery power consumption in accordance with the ETV specifications under maximum transmission efficiencies. In addition, between each scheduled delivery between workstations we must consider the precise towed weight; the floor characteristics (flat and dried concrete); the velocity profile which depends on the traveled distance; and the regenerative braking mechanism during decelerations. The initial and final runs of the ETV (from and to the SM) are also included in the emission assessment.

The ETV can load a maximum number of 100 bins, which is defined as the maximum capacity $C_{\text {max }}$, according to a previous case study in the automobile industry (Fathi et al., 2014a, 2014b). Bins weight is determined to be equal to $30 \mathrm{~kg}$, taking into consideration ergonomic risks for operators derived from hand-lifting and the towing capacity of the ETV (Limère et al., 2012). The maximum allowed speed in the plant has been considered to be $1.73 \mathrm{~m} / \mathrm{s}(6.24 \mathrm{~km} / \mathrm{h})$ which is in the range of safety velocities for internal transport in premises like factories. To reach the maximum values of regenerated energy, and according to previous optimization studies (Enang et al., 2015), we have considered an average acceleration (deceleration) value of $0.5 \mathrm{~m} / \mathrm{s}^{2}$ which is in accordance with the specifications of our electric tow vehicle. According to this, the accelerating/decelerating distances have been calculated to be equal to $3 \mathrm{~m}$.

The dynamics of the ETV is modeled to meet the standards of a heavy-duty vehicle. The energy consumption of the ETV is calculated from the traction force given the distance between visited workstations, the weight of the bins and the number of loaded bins. The traction force is calculated from the different forces involved:

$$
F=F_{\text {friction }}+F_{\text {airdrag }}+F_{\text {inertia }}
$$

Each term is described as follows: 
- The friction force is given by $F_{\text {friction }}=\mu_{s} \cdot \exp \left(-\langle v\rangle / v_{o}\right) \cdot g \cdot W$, which is in accordance with smooth modeling, responds well to the transition between static and dynamic friction forces. $\mu_{s}$ is the static friction, $\langle v\rangle$ is the average velocity, $v_{o}(=0.69 \mathrm{~m} / \mathrm{s})$ is a constant velocity calculated from the values given in Table 1 and $W$ is the total weight of the tow vehicle with cargo.

- The air-drag force is given by $F_{\text {airdrag }}=A_{d} \cdot\langle v\rangle^{2}$, where $A_{d}=0.92 \mathrm{~kg} / \mathrm{m}$ is the airdrag constant calculated from $A_{d}=\frac{1}{2} \rho \cdot C_{d} \cdot A_{F}$, being $C_{d}=0.77$ a dimensionless drag coefficient for tractor-trailers and $A_{F}\left(=1.960 .996 \mathrm{~m}^{2}\right)$ the projected frontal area according to the tow vehicle dimensions.

- The inertia force is $F_{\text {inertia }}=W \cdot R_{r} \cdot a$, where $R_{r}=1.03$ is the rotational inertia compensator factor which can substitute the rotational inertia increment in tow trains and trucks and $a$ is the acceleration of the electric tow vehicle.

Parameter values in this subsection are summarized in Table 1.

Up to this point, during acceleration displacements, the energy consumed by the battery for a maximum motor efficiency is given by:

$$
E=F \cdot d=\left[\mu_{s} \exp \left(-\langle v\rangle / v_{0}\right) g W+A_{d}\langle v\rangle^{2}+W R_{r} \cdot a\right] d
$$

Identical equations to equation (1) are used when the tow vehicle keeps a stationary velocity (with $a=0$ ) or decelerates (with negative acceleration, $-a$ ). However, during deceleration displacements, a new term $F_{r b}$ must be introduced if the regenerative braking effect is to be considered:

$$
F_{r b}=\frac{1}{2}\langle v\rangle^{2} \cdot W \cdot \eta_{b a t t}
$$

where $\eta_{\text {batt }}=0.76$ corresponds to the lead-acid battery efficiency through the regenerative braking mechanism (Helmers and Marx, 2012).

Now, the total amount of energy consumed by the battery between visited workstations is given by:

\begin{tabular}{lc}
\hline Parameters & Values \\
\hline Maximum allowed velocity (m/s) & 1.73 \\
Average acceleration (deceleration), $\pm a\left(\mathrm{~m} / \mathrm{s}^{2}\right)$ & 0.5 \\
Maximum tow vehicle capacity (no. bins) & 100 \\
Engine weight without cargo (tonnes) & 3,000 \\
Total weight of the tow vehicle, $W$ & $3,000+($ weight $/ \mathrm{bin} \times$ No. bins $)$ \\
Static friction coefficient, $\mu_{s}$ & 0.9 \\
Dynamic friction coefficient, $\mu_{d}$ & $\mu_{s} \exp \left(\frac{-\langle v\rangle}{0.69}\right) g W$ \\
Motor efficiency & 1 \\
Battery efficiency, $\eta_{\text {batt }}$ & 0.76 \\
Trapezoidal distances, $d_{ \pm a}(\mathrm{~m})$ & 3 \\
Threshold distance $(\mathrm{m})$ & 10 \\
Carbon Footprint, $C F P\left(\mathrm{~g} \mathrm{CO} \mathrm{CO}_{2}-\mathrm{eq} / \mathrm{kW} \cdot \mathrm{h}\right)$ & 500 \\
Economic cost $(€ / \mathrm{kW} \cdot \mathrm{h})$ & 0.12 \\
\hline
\end{tabular}




$$
\mathcal{E}=F_{a} \cdot d_{a}+F_{s} \cdot d_{s}+F_{-a} \cdot d_{-a}-F_{r b} \cdot d_{-a}
$$

where the forces $F_{a}, F_{s}, F_{-a}, F_{r b}$ correspond to the engine processes of acceleration, stationary velocity, deceleration and the regenerative braking during deceleration, respectively.

Finally, for the whole set of runs driven by the ETV, we are able to calculate the $\mathrm{CO}_{2}$-eq emissions considering the carbon footprint conversion (CFP) for batteries in electric vehicles charged with grid electricity (Helmers and Marx, 2012) and the economic costs according to the annual costs of electricity in the industrial sector of E.U. which are available in Eurostat (2019). Emission and economic conversion values are summarized in Table 1 as well.

\subsection{Instances generation}

Five random instances are generated for different sizes of the assembly line $(N=20,40,60)$ under a fixed daily demand. Besides, a shuffle seed produces a different daily demand $D(j)$ for each $j$-workstation and the corresponding inventory level $C(j)$ between a minimum and maximum values (Archetti et al., 2007). Initial conditions at the workstations impose a zero stock level, which means that on the very first tour the ETV finds a zero inventory level at workstations. The generation of these random instances is well supported by previous scheduling problems (Fathi et al., 2014a, 2014b; Muguerza et al., 2015) for a similar factory to guarantee a continuous production rate. Instance generation parameters are summarized in Table 2.

\section{Mathematical formulation}

To solve the assembly line feeding problem, first, we propose a MILP-model in Section 3.1, which implements a branch-and-cut algorithm using CPLEX. Despite the successful performance of the MILP-model when assessing single objectives, the outcome of the bi-objective model in terms of time processing calls for a more powerful method. In this respect, we propose a hybrid program, the HBOO-model, which is explained in Section 3.2. Then, second, we calculate emission and economic costs from the energy consumption of the ETV according to the conversion values in Table 1.

\subsection{The mixed integer linear programing model}

The MILP-model considers three different objectives in the assembly line: the number of tours, the number of stops and the inventory level. The optimal solution will determine the delivery scheduling of $i$-tours with $i=1, \ldots, T$ along an assembly line consisting of $j$-workstations with $j=1, \ldots, N$. A previous work (Fathi et al., 2014a) under the same factory conditions considered only two objectives, the number of tours and the inventory level, using a simulated annealing-based heuristic algorithm.

According to the problem instances (Section 2.2), the input data of our optimization problem are the maximum number of allowed tours, $T$; the maximum number of workstations per tour, $N$; the maximum capacity of the ETV in units of bins (100 bins for an

Table 2.

Parameter values for the generation of random instances
Inter-spacing, $d$ (meters)

5

Number of workstations, $N$

Maximum ETV capacity $C_{\max }$ (No. bins)

Bins weight (kg)
$20,40,60$

100

30 
ETV pulling 2 wagons), $C_{\text {max }}$; the daily demand assigned to each $j$-workstation, $D(j)$; and the corresponding storage capacities, $C(j)$. Furthermore, we introduce a lower bound for the minimum number of tours to be launched, $t_{l b}$, to satisfy the daily demand given the limited capacities of both the ETV and the workstations with a maximum inventory level. This value is always below the maximum number of allowed tours $T$ and is given by:

$$
t_{l b}=\max \left\{\sum_{j} \frac{\mathcal{D}(j)}{\mathcal{C}_{\text {max }}}, \max \frac{\mathcal{D}(j)}{\mathcal{C}(j)}\right\}
$$

In equation $5 \mathrm{a}$, we have formulated a multi-objective function according to a MILPproblem under a set of constraints (equations $5 \mathrm{~b}-5 \mathrm{i}$ ) and boundaries (equations $5 \mathrm{j}-5 \mathrm{l}$ ). The multi-objective function consists of three different parts, which allow us to minimize the number of tours (tours), the number of stops (stops) and the inventory level at workstations (inventory). Because each part represents a different objective function, the multi-objective function can be optimized with weighting coefficients $\left(\omega_{i}\right.$, $i=1,2,3$ ) equal to one or zero depending on the selected objects to be optimized. For instance, if the number of tours and stops are to be simultaneously minimized (tours \& stops) in equation $5 \mathrm{a}$, the weighting coefficients would be chosen to be $\omega_{1,3}=1$ and $\omega_{2}=0$. Likewise, studies on inventory-routing problems set initial weighting coefficients equal to one (Coelho et al., 2012); and also, in multicriteria decision-making problems (Cruz and Wakolbinger, 2008).

Additionally, we have notably boosted the performance of our model by introducing two binary indicator variables $y(i)$ and $s(i, j)$ (equations $5 \mathrm{~h}$ and $5 \mathrm{i}$, respectively) that act as constraints in the MILP (CPLEX) solver and have been proven to be highly effective in other MILP routing problems (Bonami et al., 2015) as it was explained in Section 1 . These binary indicator variables represent: possible tours $y(i)$ (an $i$-tour is effectively launched if $y(i)=1$, otherwise $y(i)=0$ ) and stop $s(i, j)$ (a visit to the $j$-workstation on the $i$-tour is produced if $s$ $(i, j)=1$, otherwise $s(i, j)=0)$. Finally, $z(i, j)$ represents the inventory level at a particular $j$-workstation on the $i$-tour as a continuous variable. In the constraints below, we introduce $x(i, j)$ as a dummy variable of the MILP (CPLEX) solver which represents the number of delivered bins at each $j$-workstation on each $i$-tour. In Table 3 , we present the notations of variables and constant parameters of the MILP-model. The MILP-model can be formally described as follows:

\begin{tabular}{ll}
\hline Symbol & Description in the mixed integer linear program, the MILP model \\
\hline$N$ & Number of workstations per tour $(j-$ index, $j=1, \ldots, n)$ \\
$T$ & Maximum number of allowed tours $(i$-index, $i=1, \ldots, t)$ \\
$t_{l b}$ & Minimum number of tours to satisfy the demand \\
$\omega_{k}$ & Weighting coefficients of the multi-objective function with $k=1,2,3$ \\
$y(i)$ & Binary indicator variable for tours \\
$z(i, j)$ & Inventory level at workstations after each tour \\
$s(i, j)$ & Binary indicator variable for workstations \\
$x(i, j)$ & Number of delivered bins at workstations on each tour \\
$\mathrm{C}_{\max }$ & Maximum capacity of the ETV (no. bins) \\
$C(j)$ & Storage capacity at workstations (no. bins) \\
$D(j)$ & Total demand per workstation (no. bins)
\end{tabular}

Eco-efficient management




JM2

$$
\text { (MILP) minimize } \quad \omega_{1} \sum_{i=1}^{\mathcal{T}} y(i)+\omega_{2} \sum_{i=1}^{\mathcal{T}} \sum_{j=1}^{\mathcal{N}} z(i, j)+\omega_{3} \sum_{i=1}^{\mathcal{T}} \sum_{j=1}^{\mathcal{N}} s(i, j)
$$

where $\omega_{k} \in\{0,1\}, \forall k$ subject to:

$$
\begin{gathered}
y(1)=1 \\
x(1, j)=z(1, j)>0, \forall j \\
\sum_{i} x(i, j)=\mathcal{D}(j), \forall j \\
\sum_{j} x(i, j) \leq \mathcal{C}_{\max }, \forall i \\
z(i-1, j)+x(i, j)-\frac{\mathcal{D}(j)}{t_{l b}} \leq z(i, j) \\
z(i-1, j)+x(i, j)-\frac{\mathcal{D}(j)}{\mathcal{T}} \geq z(i, j) \\
y(i) \in\{0,1\} ; y(i)=0 \Longleftrightarrow \sum_{j} s(i, j)=0, \forall i \\
s(i, j) \in\{0,1\} ; s(i, j)=0 \Longleftrightarrow x(i, j)=0, \forall i, j \\
0 \leq z(i, j) \leq \mathcal{C}(j), \forall i, j \\
0 \leq x(i, j) \leq \mathcal{C}(j), \forall i, j \\
\sum_{i} y(i) \leq \mathcal{T}, \forall j, \sum_{j} s(i, j) \leq \mathcal{N}, \forall i
\end{gathered}
$$

Initial conditions are imposed in agreement with the fact that the first tour is compulsory (equation $5 \mathrm{~b}$ ), and in this case, the delivered number of bins must fulfill equations $5 \mathrm{c}$. Also, the number of delivered bins at workstations must satisfy the daily demand $D(j)$ along with the total number of tours according to equation $5 \mathrm{~d}$. Besides, for each tour, the total number of bins carried by the ETV must not exceed the maximum train capacity $C_{\max }$ as it is shown in equation $5 \mathrm{e}$. The inventory level is controlled under equations $5 \mathrm{f}$ and $5 \mathrm{~g}$ in accordance with a deterministic production rate criteria which, for the minimum number of tours required to satisfy the daily demand as defined previously in equation (4), imposes a maximum consumption/production rate workstations of $D(j) / t_{l b}$. Meanwhile, for the 
maximum number of tours, imposes a minimum consumption/production rate workstations of $D(j) / T$. Besides, with equations $5 \mathrm{f}$ and $5 \mathrm{~g}$ we are keeping a record of the inventory level of the previous tour at workstations. Equations $5 \mathrm{~h}$ and $5 \mathrm{i}$ correspond to the binary indicator variables that account for those launched tours as $y(i)=1$ and visited workstations as $s(i, j)=$ 1 , being assigned zero values otherwise. Finally, equations $5 \mathrm{j}$ and $5 \mathrm{k}$ define the boundaries of the discrete $x(i, j)$ and continuous $z(i, j)$ variables, respectively. The corresponding upper bounds for the total number of tours and the number of workstations per tour are expressed in equation 51 .

\subsection{The hybrid bi-objective optimization and the hybrid bi-objective optimization model}

The HBOO-model developed in this study yields the optimal solution of the delivery schedule problem when the two most critical (eco-efficient) objectives, the number of tours and the number of stops, are simultaneously minimized so that the trade-off between both objectives is analyzed. The new bi-objective function has weighting coefficients $\left(\rho, \omega_{1}, \omega_{2}\right)$ as it is expressed in equation 6a. On the one hand, the pair $\left(\omega_{1}, \omega_{2}\right)$ represents the weight distribution between the two objectives so that the most important objective will be assigned the largest weight. On the other hand, the term in $\rho$, which is expressed as a linear combination of the two objectives (Equation 6a), has the role of a perturbative term that determines the degree up to which the solution search is re-directed toward the target parameter, at the knee solution of the Pareto front as explained in Section 1. The HBOOmodel can be formally described as follows:

$$
(\mathrm{HBOO}) \operatorname{minimize} \quad \omega_{1} \sum_{i=1}^{t} y(i)+\omega_{2} \sum_{i=1}^{t} \sum_{j=1}^{n} s(i, j)+\rho\left[\sum_{i=1}^{t} y(i)+\sum_{i=1}^{t} \sum_{j=1}^{n} s(i, j)\right]
$$

where $\omega_{k} \geq 0 ; \sum_{k} \omega_{k}=1, \forall k$ and $\rho \geq 0$
subject to:

$$
\sum_{i=1}^{t} y(i) \leq t^{*}
$$

Equations 5b-5i from the MILP-problem

Equations 5j-5l from the MILP-problem

Constraints and boundaries of the HBOO-model are identical to those in the MILPproblem (equations $5 \mathrm{~b}-5 \mathrm{i}$ and $5 \mathrm{j}-5 \mathrm{l}$ ) except for the new constraint in equation $6 \mathrm{~b}$, where $t^{*}$ represents the optimum number of tours and will be referred as the target parameter from now on. This constraint is characteristic of the lexicographic weighted Tchebycheff method (Steuer and Choo, 1983; Dächert et al., 2012) and allows us to guarantee the optimality of the number of tours.

We determine the values of the triad $\left(\rho, \omega_{1}, \omega_{2}\right)$ as a part of a decision-making process similar to the lexicographic weighted Tchebycheff method in (Dächert et al., 2012; SetämaaKärkkäinen et al., 2006) where parameters are adaptively chosen. As a matter of fact, by varying these parameters the sensitivity of the HBOO-the problem is tested. For this purpose, the bi-objective solutions are evaluated for an assembly line consisting of $N=40$ workstations by varying $\rho\left(\rho=110^{-03}, 110^{-02}\right)$ along with all possible combinations of weights, $\omega_{1}$ and $\omega_{2}$, each in the range $(0.1,0.9)$ with a step size of 0.2 . In addition, weights 
must fulfill the normalization condition $\omega_{1}+\omega_{2}=1$. Under these conditions, feasibility and optimality are successfully achieved for the triad values $(0.001,0.1,0.9)$ with minimum values of gap tolerances (below or equal to $5 \%$ ) and with less consuming time. From the weighting coefficients of the corresponding objectives tours and stops, the relevant role of the number of stops over the number of tours is confirmed.

In the following, the HBOO-model is described in two different phases and summarized in the flowchart of Figure 2 (notations are presented in Table 4):

- In the first phase, the Pareto front is built by varying the number of tours to predetermined values (allowed number of tours) which are ordered in a vector, $t_{P F}$. For each component of $t_{P F}$, we optimize the number of stops, which is the objective that ensures that the pool of solutions is the closest to the Pareto front. The Pareto front is defined as the inner convex boundary (Section 1) of the complete set of optimal solutions generated for each random instance. Thus, in this phase, the HBOO-model performs as a single objective optimization model by simply setting the triad parameters $\left(\rho, \omega_{1}, \omega_{2}\right)$ in equation 6 a equal to $(0,0,1)$, while the target parameter $t^{*}$ in equation $6 \mathrm{~b}$ is chosen to be equal to the corresponding component of $t_{P F}$. This phase provides the set of optimal solutions $\left(t^{*}, s^{*}\right)$ necessary to build the Pareto front. At this stage, the Pareto front is available to operators and top managers, and therefore, each candidate solution can be evaluated for alternate manufacturing scenarios.

In the second phase, which is explained next, the knee solution, $t_{k}$, is chosen to be the closest to the minimum number of allowed tours at the Pareto front. The origin of this targeting

Figure 2.

Flowchart of the HBOO-model

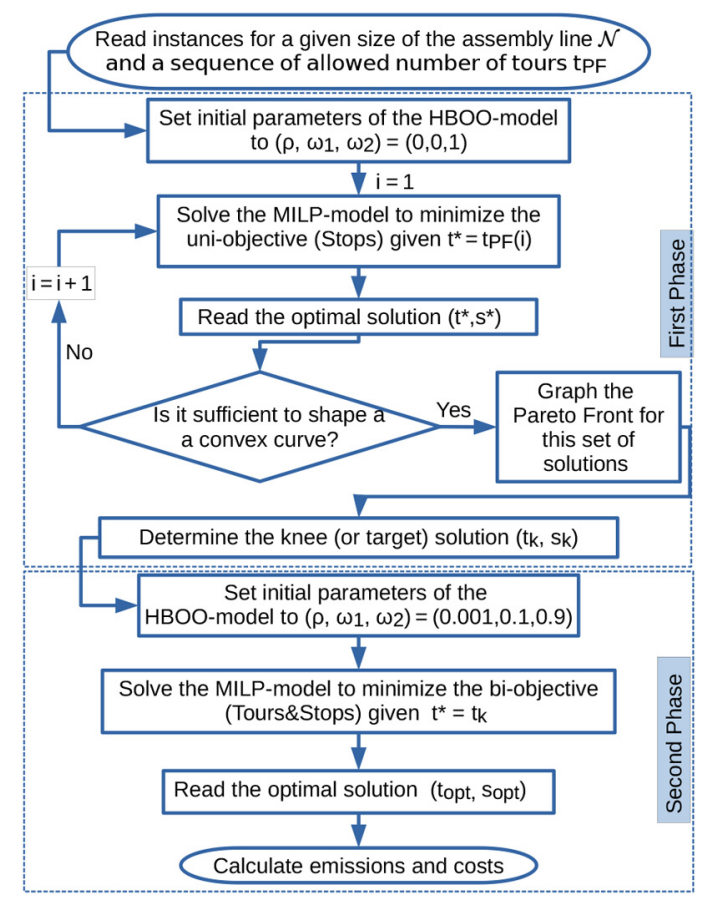


procedure must be found in the dependence between both objectives, tours and stops, which is derived from the impact that removing an entire tour has on the quantification of emissions.

Although for sufficiently long processing times, weak Pareto optimal solutions are expected to be non-dominated (Section 1), here it is important to notice that by imposing the relaxation of constraints in addition to limiting the processing times, it is possible that the final outputs could include dominated solutions. These types of solutions are called weak Pareto optimal (or weakly efficient) and as they are not removed from the final set, they are also expected to shape the Pareto front:

- In the second phase, the optimal solution $\left(t_{o p t}, s_{o p t}\right)$ is determined by setting the triad parameters $\left(\rho, \omega_{1}, \omega_{2}\right)$ in equation 6 a equal to the adaptively chosen values $(0.001$, $0.1,0.9)$ that were determined in a previous stage. Next, by setting in equation $6 \mathrm{~b}$ the target parameter equal to the knee solution obtained in the first phase $\left(t^{*}=t_{k}\right)$, the most eco-efficient solution is obtained. Because emission reduction is the aim of the present study, the optimal solution is the most convenient from an energetic perspective otherwise, in the event of a different economic or operational scenario, the management team could modify this decision by selecting a different neighboring solution at the Pareto front. On the other hand, the optimal solution provided by the HBOO-method allows decision-makers to estimate emissions and costs differences between sustainable planning and real planning.

Finally, from the optimal solution $\left(t_{o p t}, s_{o p t}\right)$ and by means of a downstream code, the total amount of energy consumed by the battery of the ETV is calculated and converted into $\mathrm{CO}_{2}$-eq emissions and economic costs (Section 2.1). In the next Section 4, we show that emissions calculated from the HBOO-optimal solutions are in agreement with the most ecoefficient assembly line management.

The algorithms were coded in the MATLAB environment with IBM ILOG CPLEX Optimization Studio V12.6.0 as the MILP solver on a personal computer.

\section{Results and discussion}

Results presented here are obtained from random instances (Section 2.2) which reproduce real conditions of the factory problem. On the grounds that the battery energy consumption responds to the dynamics of a feeding electric vehicle (as explained in Section 2.1), it must be emphasized that those parameters related to the ETV dynamics are inherent to our multioptimization function, in fact, it is the number of tours and stops that regulate travel distances and velocities distributions, while the inventory level determines the loading weight of the ETV between visited workstations.

Next, in Section 4.1, we show that energy savings obtained from the feeding electric engine model are in agreement with technical reports, in Section 4.2 we present the three-

\begin{tabular}{ll} 
Symbol & Description in the hybrid bi-objective optimization, the HBOO-model \\
\hline$\rho$ & Parameter of the perturbative term \\
$\omega_{k}$ & Weight parameters with $k=1,2,3$ \\
$t^{*}$ & Target parameter (optimum number of tours) \\
$t_{P F}$ & Sequence of the allowed number of tours \\
$\left(t_{k}, s_{k}\right)$ & Knee solution of the Pareto front \\
$\left(t_{o p t}, s_{o p t}\right)$ & Bi-objective optimal solution
\end{tabular}


dimensional Pareto fronts and in Section 4.3 we discuss the results in emissions and economic costs with a view to sustainable management. By the end of this Subsection, we also show the suitability of the developed optimization models by testing the energy efficiency against standard values.

\subsection{Testing the regenerative braking effect of the electric tow vehicle}

Prior to the optimization analysis of the whole set of instances, we present the results on the effect of the regenerative braking over an assembly line of $N=40$ workstations, as a benchmark. In Table 5, results in energy savings when different objectives are minimized are presented. Energy savings are calculated from the results with regenerative braking with respect to those without regenerative braking. Notice that the higher percentages in energy savings correspond to the most economically profitable solutions so that the most eco-efficient solution corresponds to minimizing simultaneously the number of tours and stops, tours\&stops. Percentages in energy savings are in agreement with previous studies (Helmers and Marx, 2012).

In Figure 3, we present three-dimensional diagrams of emission levels during the feeding process. The assembly line of this figure consists of 40 workstations with an inter-spacing of $d=5 \mathrm{~m}$, where both, the number of tours and stops, are simultaneously minimized. The $z$ axis corresponds to the levels of $\mathrm{CO}_{2}$-eq emissions (in units of $\mathrm{g}$ ) while the number of tours and workstations is represented on the horizontal XY-plane. Cold colors correspond to low emission values, while hot colors correspond to high emission values. Results with the regenerative braking effect of the ETV are shown in Figure 3(a) in contrast to Figure 3(b) (without regenerative braking). Differences in emission levels are revealed if we simply compare the colored bar ranges. These differences are quantified in the corresponding Table 5, where it can be observed that the energy and economic savings corresponding to the regenerative braking effect are of the order of $60 \%$ which is in accordance with previous reports (IEA, 2007). From now on, the discussion focuses on the results with regenerative braking.

Table 5.

Energy savings under the regenerative braking effect

\section{Objective}

Tours\&stops

Stops

Tours

Inventory
Energy savings $(N=40)$

\section{$63 \%$ \\ $55 \%$ \\ $36 \%$ \\ $4 \%$}

Figure 3.

$\mathrm{CO}_{2}$-eq emissions along an assembly line for $N=40$ workstations: with regenerative braking in (a) and without in (b)

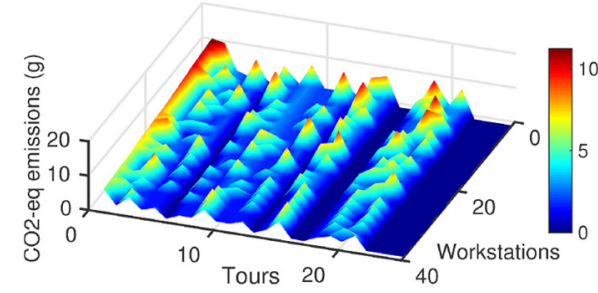

(a)

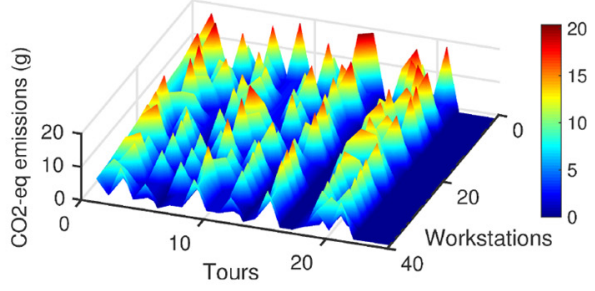

(b) 


\subsection{The three-dimensional Pareto fronts}

In Figure 4, we show typical three-dimensional Pareto fronts for $N=40$ and 60 workstations. These Pareto fronts are built calculating the corresponding $\mathrm{CO}_{2}$-eq emissions (z-axis) from the set of optimal solutions $\left(t^{*}, s^{*}\right)$, which shape a convex Pareto front on the xyplane, obtained in the first phase of the HBOO-method (Section 3.2). Minimum emissions (black circles) correspond to the optimal solutions $\left(t_{o p t}, s_{o p t}\right)$ generated in the second phase of the HBOO-method by introducing the target solution $t_{k}$ (knee solution of the Pareto front), as it was explained in Section 3.2. Notice that, these optimal solutions are averaged over each set of instances. In this figure, we observe that although there is a quasi-linear increase in emissions with respect to the number of tours, for $N=60$ there is a discontinuity in the Pareto front above 24 tours and that subsequent solutions appear to be slightly more scattered. The integer nature of the variables (the number of tours and stops) and the existence of weakly Pareto optimal solutions generated by the lexicographic optimization approach may underlie this fact as it has been previously reported in Farmani et al. (2007).

\subsection{The mixed integer linear programing and hybrid bi-objective optimization models:}

annual emissions and economic costs

In the following, we infer general eco-efficient measures to be implemented in similar assembly-line plants by comparing the effects of minimizing single objectives (tours, stops

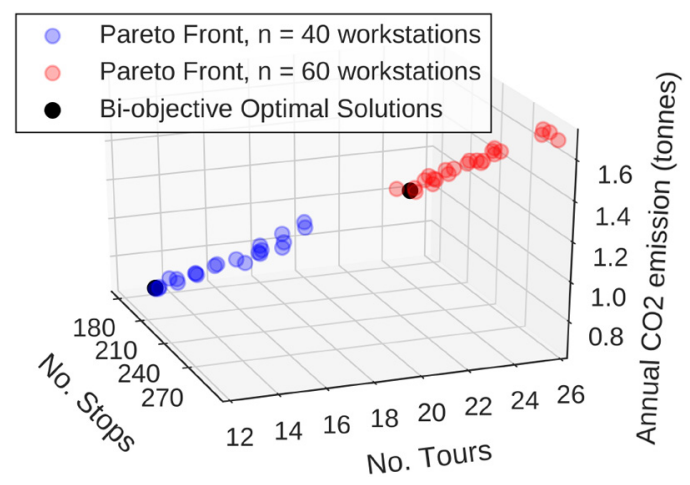

Figure 4

Three-dimensional

Pareto fronts and optimal solutions (black circles)

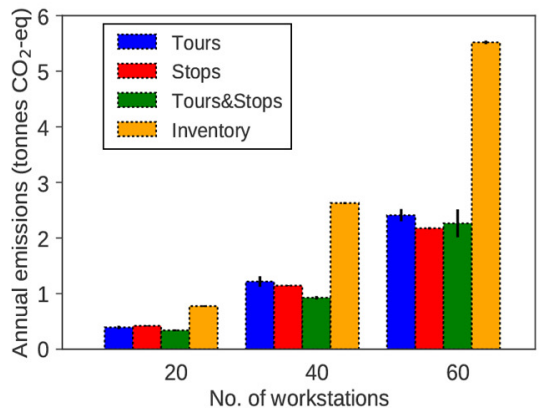

(a)

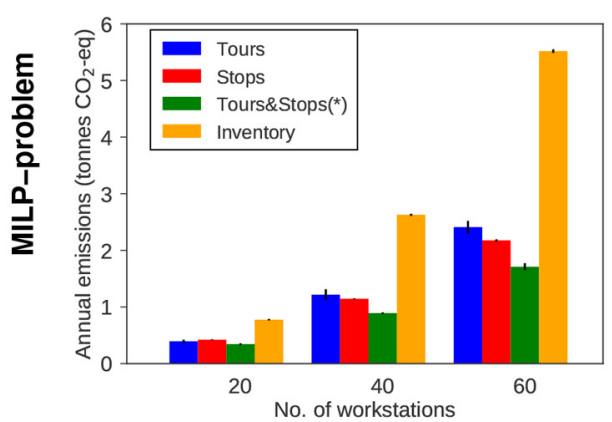

(b)

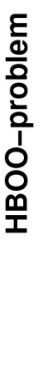

Figure 5. $\mathrm{CO}_{2}$-eq emissions versus the number of workstations in the assembly line when different objectives are minimized 
and inventory), and the bi-objective (tours\&stops). These results are in agreement with audited reports in the transport sector and manufacturing industry (Haven and Gutin, 2015; EPA, 2015) as it will be shown as a concluding remark in this Subsection.

In Figure 5, the average values of annual $\mathrm{CO}_{2}-$ eq emissions are calculated for each set of instances by minimizing single objectives and the bi-objective as indicated. Error bars are calculated as the standard deviation of optimal solutions obtained over each set of random instances. In Figure 5(a), annual $\mathrm{CO}_{2}$-eq emissions using the MILP-model are shown in comparison to emissions in Figure 5(b) using the HBOO-model. From the energy consumption of the ETV, we also calculate the annual economic costs with equivalent results to those of emissions in the sense that relative ratios between different objectives are identical (Figure 6). From the energetic and economic costs in these figures, it is found that minimizing the objective inventory doubles and even, in certain cases, triples the costs. In contrast, the most competitive solution is obtained when the bi-objective tours\&stops is minimized using the HBOO-model. If we compare emissions (and costs) when minimizing single objectives relative to minimizing the bi-objective tours\&stops using the HBOOmodel, we can say that the manufacturer would incur additional costs of the order of the percentage values summarized in Table 6, which quantitatively supports the discussion above.

Next, we analyze the annual $\mathrm{CO}_{2}$-eq emissions as the size of the assembly line increases in terms of $N$ [Figure 5(a) and 5(b)]:

- For a number of workstations equal to $N=20$, the bi-objective tours\&stops is the most eco-efficient solution. Nevertheless, when these objectives are minimized as single-objective, the MILP-model provides competing levels of emissions. In the previous study, we had checked this very same emission bar distribution for an assembly line consisting of fewer workstations $(N=10)$. According to this, for $N \leq$

Figure 6.

Economic costs versus the number of workstations in the assembly line when different objectives are minimized

\section{Table 6.}

Relative energetic (economical) costs with respect to minimizing tours\&stops

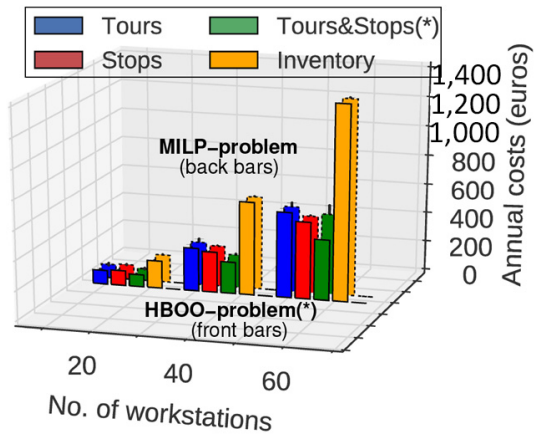

\begin{tabular}{lcrr}
\hline$N$-workstations & \multicolumn{1}{c}{40} & \multicolumn{1}{c}{60} \\
\hline Minimizing tours (\%) & 14.45 & 36.61 & 41.00 \\
Minimizing stops (\%) & 22.60 & 28.36 & 27.25 \\
Minimizing inventory (\%) & 126.11 & 194.88 & 222.73 \\
\hline
\end{tabular}


20 optimal solutions when minimizing tours appear to be more eco-efficient than minimizing stops.

- As the size of the assembly line is increased, $N=40$ workstations, we observe that although similar ratios between different bars are obtained, the rivalry between the single-objectives tours with respect to stops now happens to favor the latter as the most eco-efficient solution [Figure 5(a)]. Even though the bi-objective tours\&stops still remains the most eco-efficient solution.

- In Figure 5(a), as the number of workstations is increased from $N=40$ to 60, we find that minimizing stops is still more eco-efficient than minimizing tours. However, in the same figure, it is observed that the bi-objective tours\&stops optimization using the MILP-model (dotted green bars) yields higher levels of emissions than stops for $N=60$ workstations in comparison to $N=40$. Thus, a crossover is found between $N=40$ and 60 workstations when the MILP-model is implemented if we compare the results when minimizing stops with tours\&stops. In contrast, when the HBOOmodel is implemented to solve the bi-objective tours\&stops problem [Figure 5(b)], emissions from $N=40$ up to $N=60$ workstations show no crossover between stops and tours\&stops.

The bi-objective optimization results for $N=40$ and 60 workstations can be quantitatively compared from results in Table 7 (ETV). Here, we can observe that the relative error when using the HBOO-the model has decreased by $52 \%$ and $74 \%$, respectively. Then, as expected, in the case of $N=20$ workstations, there are no significant differences when the HBOO-method is used. Additionally, in the same table, we show the optimized emissions produced by the ETV in comparison with the calculated emissions produced by an oil-fueled heavy-duty-vehicle (HDV). The estimation of emissions produced by the HDV (Haven and Gutin, 2015; EPA, 2015), are calculated for the minimum number of tours (obtained when minimizing tours) and average cargo weight of 3 tonnes. It should be stressed that, in the manufacturing industry, engines specific to material handling consume of the order of $10 \%$ of the manufacturing electricity used (DOE, 2002; IEA, 2007). In the case of the USA and for the entire volume of manufacturing industries, this percentage would correspond to emissions of the order of $210^{6}$ tonnes $\mathrm{CO}_{2} /$ year and costs less than $10 \mathrm{~m}$ dollars (DOE, 2002), from which feeding vehicles would contribute to a small amount. Be that as it may if we put into perspective the optimized results of the feeding ETV against the HDV for a number of workstations larger than 20, emissions produced by the ETV in contrast to the HDV are significantly half-reduced which respond well to the scope of our research.

\section{Conclusions and future studies}

In this paper, we have determined those decision variables that play a decisive role in diminishing the carbon footprint and the economic costs in the scheduling problem of an automobile assembly line. Instances are randomly generated for a predetermined demand

\begin{tabular}{llll}
\hline$N$-workstations & \multicolumn{1}{c}{20} & \multicolumn{1}{c}{40} & 60 \\
\hline ETV(MILP-model) & $0.340 \pm 0.015$ & $0.928 \pm 0.030$ & $2.263 \pm 0.251$ \\
ETV (HBOO-model) & $0.343 \pm 0.016$ & $0.891 \pm 0.014$ & $1.709 \pm 0.064$ \\
HDV & 0.328 & 1.423 & 3.120
\end{tabular}

Table 7.

Emissions (tonnes $\mathrm{CO}_{2}$-eq) by electric (ETV) and oil-fueled (HDV) vehicles when tours\&stops are minimized 
according to a real factory problem. The objective functions of our feeding problem are given by the number of tours, the number of stops and the inventory level at workstations. In the first place, to minimize the corresponding multi-objective optimization problem, we propose a MILP-model for different sizes of the assembly line. However, a bi-objective optimization problem naturally arises from the competing emission levels of the two most eco-efficient objectives: the number of tours and stops. In this context, a hybrid bi-objective optimization HBOO-model is developed using a lexicographic weighted Tchebycheff approach with adaptive values of the weighting coefficients. Finally, a downstream code is generated to calculate emissions levels and economic costs from the battery energy consumption of a feeding ETV. The fact of analyzing the energy consumption by means of a downstream code without using an energy function in the multi-objective optimization function responds well to the fact that, in our problem, it is the dynamics of the ETV and the interrelated dissipation processes that drain energy from the battery.

The most significant result of our study is that, for any size of the assembly line, the most eco-efficient strategy is always to minimize the bi-objective problem given by the number of tours and stops using the HBOO-method. Besides, in contrast to the MILP-model, the HBOOmodel has successfully proven feasibility and optimality with a minor impact on the gaps of the branch-and-cut algorithm and on the processing times. This result is of utmost importance whenever operational managers or other decision-makers, must design new strategies or analyze the need for new investments to diminish the environmental impact of the assembly line performance. This is because of the flexibility of the HBBO-model which is built in twophases and whose results are available independently: the first phase provides the Pareto front from which neighboring solutions to the optimal solution can be evaluated for alternate scheduling, while the second phase provides the most eco-efficient scheduling.

By comparison, results concerning single objectives show that the most eco-efficient solution is always to minimize the number of stops, except for assembly lines consisting of 20 or fewer workstations, in which case minimizing the number of tours is more efficient than minimizing the number of stops as expected. Meanwhile, the less eco-efficient management always corresponds to the minimization of the inventory level which at the very least doubles costs.

Moreover, our results are validated by the estimation of energy savings from the dynamics of an ETV, with regenerative braking effect, which are in agreement with audited reports. Furthermore, it is shown that emissions calculated in our green assembly line are half-reduced in comparison to a feeding oil-fueled vehicle under the same conditions. As a consequence, the HBOO-model is suitable to advise decision-makers to promote new measures in green in-house logistics, specifically in feeding and inventory-routing problems whenever motor-driven engines are used. Likewise, these results encourage the transition of oil-fueled feeding vehicles to electric ones in those manufacturing industries eager to implement eco-efficient initiatives.

All in all, these results support scheduling autonomous feeding electric vehicles according to the proposed bi-objective optimization model, the HBOO-model, as the best strategy to improve the eco-efficiency management of an assembly line in the automobile industry and by extension to other assembly lines in the aerospace, civil engineering and renewable energy industries to assess eco-efficient policies.

In this direction, and given that the proposed model allows analysts to assess the performance of a green assembly line and decision-makers to have useful decision aid, further work should consider, on the one hand, developing a contingency plan using the HBOO-model over instances that would reproduce urgent adverse in-plant situations, for example, in the event of a supplier failure or the detection of quality defects in the production. Then, also, over longer periods of time, for example, under the circumstance that 
the production rate could be affected by a change in the customer's tastes. On the other hand, and from a managerial perspective, to further improve the eco-efficiency of the assembly line performance on a daily basis, a more friendly code of the HBOO-model would contemplate the re-evaluation of the weighting coefficients according to recent production rates.

\section{Note}

1. https://developer.ibm.com/academic/

\section{References}

Alavidoost, M.H., Tarimoradi, M. and Zarandi, M.H.F. (2018), "Bi-objective mixed-integer nonlinear programming for multi-commodity tri-echelon supply chain networks", Journal of Intelligent Manufacturing, Vol. 29 No. 4, pp. 809-826.

Alexander, A., Walker, H. and Naim, M. (2014), "Decision theory in sustainable supply chain management: an international journal: a literature review", Supply Chain Management: An International Journal, Vol. 19 No. 5/6, pp. 504-522.

Andres, C., Miralles, C. and Pastor, R. (2008), "Balancing and scheduling tasks in assembly lines with sequence-dependent setup times", European Journal of Operational Research, Vol. 187 No. 3, pp. 1212-1223.

Araque, J., Kudva, G., Morin, T. and Pekny, J. (1994), “A branch-and-cut algorithm for vehicle routing problems", Annals of Operations Research, Vol. 50 No. 1, pp. 37-59.

Archetti, C., Bertazzi, L., Laporte, G. and Speranza, M. (2007), "A branch-and-cut algorithm for a vendor-managed inventory-routing problem”, Transportation Science, Vol. 41 No. 3, pp. 382-391.

Azmi, N.I.L.M., Ahmad, R. and Zainuddin, Z.M. (2017), "MILP model for integrated balancing and sequencing mixed-model two-sided assembly line with variable launching interval and assignment restrictions", Journal of Physics: Conference Series, Vol. 890, p. 012107.

Barcos, L., Rodríguez, V., Álvarez, M. and Robusté, F. (2010), "Routing design for less-than-truckload motor carriers using ant colony optimization", Transportation Research Part E: Logistics and Transportation Review, Vol. 46 No. 3, pp. 367-383.

Bazan, E., Jaber, M. and Zanoni, S. (2015), "Supply chain models with greenhouse gases emissions, energy usage and different coordination decisions", Applied Mathematical Modelling, Vol. 39 No. 17, pp. 5131-5151.

Benjaafar, S., Li, Y. and Daskin, M. (2013), "Carbon footprint and the management of supply chains: insights from simple models", IEEE Transactions on Automation Science and Engineering, Vol. 10 No. 1, pp. 99-116.

Bierwirth, C., KirschsteinDirk, T. and Sackmann, D. (2019), Logistics Management, Springer, Cham.

Bonami, P., Lodi, A., Schweiger, J. and Tramontani, A. (2019), "Solving quadratic programming by cutting planes", SIAM Journal on Optimization, Vol. 29 No. 2, pp. 1076-1105.

Bonami, P., Lodi, A., Tramontani, A. and Wiese, S. (2015), "On mathematical programming with indicator constraints", Mathematical Programming, Vol. 151 No. 1, pp. 191-223.

Boysen, N., Fliedner, M. and Scholl, A. (2007), "A classification of assembly line balancing problems", European Journal of Operational Research, Vol. 183 No. 2, pp. 674-693.

Coelho, L., Cordeau, J. and Laporte, G. (2012), "Consistency in multi-vehicle inventory-routing", Transportation Research Part C: Emerging Technologies, Vol. 24, pp. 270-287.

Cruz, J. and Wakolbinger, T. (2008), "Multiperiod effects of corporate social responsibility on supply chain networks, transaction costs, emissions, and risk", International Journal of Production Economics, Vol. 116 No. 1, pp. 61-74. 
Dächert, K., Gorski, J. and Klamroth, K. (2012), "An augmented weighted tchebycheff method with adaptively chosen parameters for discrete bicriteria optimization problems", Computers and Operations Research, Vol. 39 No. 12, pp. 2929-2943.

Das, C. and Jharkharia, S. (2018), "Low carbon supply chain: a state-of-the-art literature review", Journal of Manufacturing Technology Management, Vol. 29 No. 2, pp. 398-428.

DOE2002 IIchenas03.cadmus.comlsmarteditlNormalizationUININPROCESS 18 . "United States industrial electric motor systems market assessment opportunities. Xenergy report, US Department of energy office of energy efficiency and renewable energy office of industrial technologies", available at www.energy.gov/sites/prod/files/2014/04/f15/mtrmkt.pdf(accessed 23 September 2019).

Ehrgott, M. (2005), Multicriteria Optimization, Springer-Verlag, Berlin Heidelberg.

Emmerich, M.T.M. and Deutz, A.H. (2007), “Test problems based on lamé superspheres", In: Evolutionary Multi-Criterion Optimization, Vol. 4403. Springer, Berlin, Heidelberg, pp. 922-936.

Enang, W., Bannister, C., Brace, C. and Vagg, C. (2015), "Modelling and heuristic control of a parallel hybrid electric vehicle", Journal of Automobile Engineering, Vol. 229 No. 11, pp. 1494-1513.

EPA2015 Ilchenas03.cadmus.com/smarteditlNormalizationUINYINPROCESS|22. "Greenhouse gas emissions and fuel efficiency standards for medium and heavy-duty engines and vehicles. Proposed rules, federal register, US Environmental protection agency and department of transportation", available at: www.govinfo.gov/content/pkg/FR-2015-07-13/pdf/2015-15500.pdf (accessed 24 September 2019).

Eurostat2019 IIchenas03.cadmus.comlsmartedit|Normalization|INIINPROCESS123. "Electricity price statistics. Tech. rep., statistics explained", available at: https://ec.europa.eu/eurostat/statisticsexplained/pdfscache/45239.pdf(accessed 23 September 2019).

Farmani, R., Savic, D.A. and Walters, G.A. (2007), "On convergence of multi-objective pareto front: perturbation method", In: Evolutionary Multi-Criterion Optimization, Vol. 4403, Springer, Berlin, Heidelberg, pp. 443-456.

Fathi, M., Rodríguez, V. and Álvarez, M. (2014b), “A novel memetic ant colony optimization-based heuristic algorithm for solving the assembly-line part feeding problem", The International Journal of Advanced Manufacturing Technology, Vol. 75 No. 1-4, pp. 629-643.

Fathi, M., Álvarez, M., Hassani, F. and Rodríguez, V. (2014a), “A multiobjective optimization algorithm to solve the part feeding problem in mixed-model assembly-lines", Mathematical Problems in Engineering, Vol. 2014, p. 654053.

Fathi, M., Fontes, D., Urenda Moris, M. and Ghobakhloo, M. (2018), "Assembly line balancing problem: a comparative evaluation of heuristics and a computational assessment of objectives", Journal of Modelling in Management, Vol. 13 No. 2, pp. 455-474.

Figueira, J., Greco, S. and Ehrgott, M. (2005), Multiple Criteria Decision Analysis, Springer Science and Business Media.

Gharaei, A., Reza Pasandideh, S.H. and Arshadi Khamseh, A. (2017), "Inventory model in a four-echelon integrated supply chain: Modeling and optimization", Journal of Modelling in Management, Vol. 12 No. 4.

Haven, P. and Gutin, O. (2015), "Vehicle efficiency and emissions standards. Fact sheet”, Environmental and Energy Study Institute, available at: www.eesi.org/papers(accessed 24 September 2019).

Helmers, E. and Marx, P. (2012), "Electric cars: technical characteristics and environmental impacts", Environmental Sciences Europe, Vol. 24 No. 1, p. 14, available at: www.enveurope.com/content/ 24/1/14

Huang, L. and Yang, J. (2019), "Location-distribution of cruise ship supply logistics distribution Centre considering time window”, Systems Science and Control Engineering, Vol. 7 No. 1, pp. 338-345.

IEA2007 IIchenas03.cadmus.com|smartedit|Normalization|ININPROCESSI33. "Tracking industrial energy efficiency and CO2 emissions. Tech. rep., the international energy agency", available at: 
www.iea.org/publications/freepublications/publication/tracking_emissions.pdf(accessed September 2019).

Jin, L., Chen, P. and Liu, Y. (2014), "An analysis of regenerative braking and energy saving for electric vehicle with in-wheel motors", International Journal of Control and Automation, Vol. 7 No. 12 , pp. 219-230.

Kähkönen, A.K. (2020), "Sustainable supply management practices: making a difference in a firm's sustainability performance", Supply Chain Management: An International Journal, Vol. 23 No. 6, pp. 518-530.

Keskin, M., Laporte, G. and Çatay, B. (2019), "Electric vehicle routing problem with time-dependent waiting times at recharging stations", Computers and Operations Research, Vol. 107, pp. 77-94.

Kopfer, H.W. and Kopfer, H. (2013), "Emissions minimization vehicle routing problem in dependence of different vehicle classes", Dynamics in Logistics.Lecture Notes in Logistics, Springer, Berlin, Heidelberg.

Latha Shankar, B., Basavarajappa Rajeshwar, S. and Kadadevaramath, S. (2012), "Bi-objective optimization of distribution scheduling using mopso optimizer", Journal of Modelling in Management, Vol. 7 No. 3, pp. 304-327.

Lee, J. (2005), "Rotating inertia impact on propulsion and regenerative braking for electric motor driven vehicles", Ph.D. thesis, Virginia Polytechnic Institute and State University.

Limère, V., Landeghem, H., Goetschalckx, M., Aghezzaf, E. and McGinnis, L. (2012), "Optimising part feeding in the automotive assembly industry: deciding between kitting and line stocking", International Journal of Production Research, Vol. 50 No. 15, pp. 4046-4060.

Liu, J., Srai, S. and Evans, S. (2016), "Environmental management: the role of supply chain capabilities in the auto sector", Supply Chain Management: An International Journal, Vol. 21 No. 1, pp. 1-19.

Muguerza, M., Briand, C., Jozefowiez, N., Ulrich, S., Rodríguez, V. and Urenda, M. (2015), “A mass-flow MILP formulation for energy-efficient supplying in assembly-lines", In: Multidisciplinary International Scheduling Conference: Theory and Applications. The MISTA Conference Series, pp. 236-239.

Petridis, K., Dey, P.K. and Emrouznejad, A. (2017), “A branch and efficiency algorithm for the optimal design of supply chain networks”, Annals of Operations Research, Vol. 253 No. 1, pp. 545-571.

Rodríguez, V., Álvarez, M. and Barcos, L. (2007), "Hub location under capacity constraints", Transportation Research Part E: Logistics and Transportation Review, Vol. 43 No. 5, pp. 495-505.

Sadrnia, A., Ismail, N., Zulkifli, N., Ariffin, M.K.A., Nezamabadi, H. and Mirabi, H. (2013), "A multiobjective optimization model in automotive supply chain networks", Mathematical Problems in Engineering, Vol. 2013, pp. 1-10.

Salhi, S. and Imran, A. (2014), "The multi-depot vehicle routing problem with heterogeneous vehicle fleet: Formulation and a variable neighborhood search implementation", Computers and Operations Research, Vol. 52, pp. 315-325.

Sawaragi, Y., Nakayama, H. and Tanino, T. (1985), Theory of Multiobjective Optimization. Mathematics in Science and Engineering, Academic Press.

Scholl, A., Boysen, N. and Fliedner, M. (2013), "The assembly line balancing and scheduling problem with sequence-dependent setup times: problem extension, model formulation and efficient heuristics", Or Spectrum, Vol. 35 No. 1, pp. 291-320.

Setämaa-Kärkkäinen, A., Miettinen, K. and Vuori, J. (2006), "Best compromise solution for a new multiobjective scheduling problem", Computers and Operations Research, Vol. 33 No. 8, pp. 2353-2368.

Shijin, W. and Yulun, W. (2015), "A genetic algorithm for energy minimization vehicle routing problem. In: 2017 international conference on service systems and service management. Services systems and services management", ICSSSM, International Conference on, IEEE, pp. 1-5. 
Srivastava, S.K. (2007), "Green supply chain management: a state of the art literature review", International Journal of Management Reviews, Vol. 9 No. 1, pp. 53-80.

Steuer, R.E. and Choo, E.U. (1983), "An interactive weighted tchebycheff procedure of multiple objective programming”, Mathematical Programming, Vol. 26 No. 3, pp. 326-344.

Toth, P. (2008), "An integer linear programming local search for capacitated vehicle routing problems. The vehicle routing problem: latest advances and new challenges", Operations Research/ Computer Science Interfaces, vol 43. Springer, Boston, MA.

Wang, G., Gunasekaran, A., Ngai, E. and Papadopoulos, T. (2016), "Big data analytics in logistics and supply chain management: Certain investigations for research and applications", International Journal of Production Economics, Vol. 176, pp. 98-110.

Wassan, N.A., Wassan, N., Nagy, G. and Salhi, S. (2017), "The multiple trip vehicle routing problem with backhauls: formulation and a two-level variable neighbourhood search", Computers and Operations Research, Vol. 78, pp. 454-467.

$\mathrm{Xu}, \mathrm{G} ., \mathrm{Li}, \mathrm{W} ., \mathrm{Xu}, \mathrm{K}$. and Song, Z. (2011), “An intelligent regenerative braking strategy for electric vehicles”, Energies, Vol. 4 No. 9, pp. 1461-1477.

Yu, F.V. and Jewpanya, P. (2016), "Open vehicle routing problem with cross-docking”, Computers and Industrial Engineering, Vol. 94, pp. 6-17.

Zhen, L. (2017), “A bi-objective model on multiperiod green supply chain network design”, IEEE Transactions on Systems, Man, and Cybernetics: Systems, Vol. 50 No. 3, pp. 1-14.

Zhou, B.H. and Shen, C.Y. (2018), "Multi-objective optimization of material delivery for mixed model assembly lines with energy consideration”, Journal of Cleaner Production, Vol. 192, pp. 293-305.

\section{Corresponding author}

Montserrat-Ana Miranda can be contacted at: montse@alumni.unav.es

For instructions on how to order reprints of this article, please visit our website: 